\title{
Inland harmful algal blooms (HABs) modeling using internet of things (IoT) system and deep learning
}

\author{
Do Hyuck Kwon ${ }^{1}$, Seok Min Hong ${ }^{1}$, Ather Abbas ${ }^{1}$, JongCheol Pyo ${ }^{2}$, Hyung-Kun Lee ${ }^{3}$, Sang-Soo \\ Baek $^{1 \dagger}$, Kyung Hwa Cho ${ }^{1 \dagger}$
}

${ }^{1}$ School of Urban and Environmental Engineering, Ulsan National Institute of Science and Technology, Ulsan, 44919, Republic

of Korea

${ }^{2}$ Center for Environmental Data Strategy, Korea Environment Institute, Sejong 30147, Republic of Korea

${ }^{3}$ ICT Materials and Components Research Laboratory, Electronics and Telecommunications Research Institute (ETRI),

Daejeon, Republic of Korea

Received June 13, $2021 \quad$ Revised October 19, $2021 \quad$ Accepted January 20, 2022

\begin{abstract}
Harmful algal blooms (HABs) have been frequently occurred with releasing toxic substances, which typically lead to water quality degradation and health problems for humans and aquatic animals. Hence, accurate quantitative analysis and prediction of HABs should be implemented to detect, monitor, and manage severe algal blooms. However, the traditional monitoring required sufficient expense and labor while numerical models were restricted in terms of their ability to simulate the algae dynamic. To address the challenging issue, this study evaluates the applicability of deep learning to simulate chlorophyll-a (Chl-a) and phycocyanin (PC) with the internet of things (IoT) system. Our research adopted LSTM models for simulating Chl-a and PC. Among LSTM models, the attention LSTM model achieved superior performance by showing 0.84 and $2.35(\mu \mathrm{g} / \mathrm{L})$ of the correlation coefficient and root mean square error. Among preprocessing methods, the $\mathrm{z}$-score method was selected as the optimal method to improve model performance. The attention mechanism highlighted the input data from July to October, indicating that this period was the most influential period to model output. Therefore, this study demonstrated that deep learning with IoT system has the potential to detect and quantify cyanobacteria, which can improve the eutrophication management schemes for freshwater reservoirs.
\end{abstract}

Keywords: Attention mechanism, Deep learning, Harmful algal blooms (HABs), Internet of things (IoT), Water quality

\footnotetext{
(2) $(1) \Theta$

This is an Open Access article distributed under the terms of the Creative Commons Attribution Non-Commercial License (http://creativecommons.org/licenses/by-nc/3.0/) which permits unrestricted non-commercial use, distribution, and reproduction in any medium, provided the original work is properly cited.
}

Corresponding Author

E-mail: khcho@unist.ac.kr; kbcqr12@unist.ac.kr Tel: +82-52-217-2829; +82-52-217-2886 Fax: +82-52-217-2819; +82-52-217-2819 ORCID: 0000-0003-3157-0295 


\section{1. Introduction}

2 The outbreak of harmful algal blooms (HABs) adversely affected water quality in rivers and

3 lakes [1]. HABs have been frequently reported at tglobal scale according to rapid urbanization

4 and global climate change [2,3]. The algae can release toxic substances, which typically lead to

5 water quality degradation and health problems for humans and aquatic animals [4]. Since the

6 construction of a multi-functional dam and weir in major rivers of South Korea, the country has

7 experienced cyanobacteria outbreaks, which release microcystin, a toxic substance that

8 negatively affects the human body [5]. In particular, Daechung reservoir in South Korea has

9 annually endured the outbreak of HABs due to the inflowing massive nutrient and warm water

10 [6]. Hence, an accurate quantitative and qualitative analysis of HABs via monitoring should be

11 implemented to detect, monitor, and regulate severe algal blooms [7-9].

South Korea equips the algal alert system to monitor water quality for securing public

13 health and drinking water. This monitoring system has weekly measured water quality related to

14 HABs and notifies the government agency of the observation [10]. However, the weekly

15 monitoring cannot identify the instant change of HABs because the dynamic of HABs has high

16 variation and uncertainty [11]. In addition, persistent HABs monitoring is time consuming,

17 costly, and labor intensive [12]. Recently, the internet of things (IoT) platform including

18 detection sensors and wireless network has been proposed as a promising monitoring technique,

19 since it is capable of receiving the real-time data of water quality [13, 14]. Hu et al. [15] acquired

20 water quality data through the real-time monitoring using the detection sensors and the mobile

21 online servers. They have collected real-time data such as water temperature, dissolved oxygen,

22 salinity, and $\mathrm{pH}$ level. Although the real-time monitoring system can be useful to identify the

23 deterioration of water quality, few studies have applied this technique to HABs monitoring. 
Given the limited resources, understanding HABs via simulation could be useful to

control the outbreak of algae [16]. The simulation of water quality through modeling regards important to determine the policy decisions for effective water resources management. Previous studies have developed numerical-based water quality models to understand the dynamics of algae, including the Environmental Fluid Dynamics Code (EFDC), Soil Water Assessment Tool (SWAT), and CE-QUAL-W2 [17-19]. However, these models were restricted in terms of their ability to simulate algal dynamics $[5,20,21]$. Additionally, these models have a challenging issue regarding the complexity of HAB dynamics depending on multiple physical, chemical, and biological system effects $[10,22]$. To address this challenge, the data-driven model has been introduced as an alternative approach to predict water quality and HABs by learning non-linear mathematical relations between input and output data [23]. Specifically, long Short-Term Memory (LSTM) has a considerable advantage in the time-series data [24]. Baek et al. [25] simulated the water level, total nitrate (TN), total phosphorus (TP), and total organic carbon (TOC) using LSTM. Zhang et al. [26] utilized the LSTM model to predict the water level in the urban drainage system. However, these models are limited to explain the correlation between input and output variables, and the observation [27, 28]. Data preprocessing also has an important role in machine learning and deep learning algorithms, and proper data preprocessing is compulsory for achieving better model performance [29]. Shen et al. [30] demonstrated that it is necessary to use the preprocessing method for managing big data prior to the application of data-driven models.

Hence, we aim to evaluate the applicability of deep learning to simulate chlorophyll-a (Chl-a) and phycocyanin (PC) concentrations with real-time monitoring in Daechung reservoir, South Korea. Chl-a and PC are the proxy indicator of the algal biomass, Chl-a is an indicator of 
47 phytoplankton biomass and PC is an accessory pigment of cyanobacteria [31, 32]. Our research 48 adopted state-of-the-art data-driven models, attention LSTM. the attention mechanism is the 49 overcoming approach with explainability by analyzing the features of attention weight [33]. In 50 this regard, the main objectives of our research were to: (1) conduct HABs monitoring via IoT

51 system, (2) simulate Chl-a and PC concentrations using LSTM models, (3) evaluate the model 52 performance depending on the data preprocessing method, and (4) interpret the model result 53 through attention weights of the model.

\section{Material and Methods}

\subsection{Study Area and Data Acquisition}

57 Daechung reservoir is located in upstream of Geum River, South Korea (N 36.35-36.52, E 58 127.48-127.60) (Fig. 1). This reservoir has supplied water to nearby cities (e.g. Daejeon and 59 Chungju) for agricultural, domestic, and industrial use [34]. The water surface area and storage 60 capacity are $72.8 \mathrm{~km}^{2}$ and $1,490 \times 10^{6} \mathrm{~m}^{3}$, respectively [35]. This site has the frequent 61 occurrences of HAB from summer to late autumn [36]. The HABs by cyanobacteria have been 62 annually reported during this season as regular events [37]. We measured Chl-a, PC, and seven 63 water quality variables at two stations: Hoenam and Chusori. Hoenam is a transition zone that 64 flows into the reservoir in the mainstream of the Geum River [38, 39]. Chusori has inflow from 65 excessive anthropogenic sources including the sewage treatment water and fertilizers [40]. The 66 monitoring was conducted from January to December in 2020. TN and TP were obtained by 67 Ministry of Environment [41]. Meteorological data were acquired from near weather stations 68 (e.g., Secheon (N 36.35-36.52, E 127.48-127.60) and Cheongnamdae (N 36.35-36.52, E 127.48- 
127.60)) [42]. Daily inflow and outflow of the reservoir were measured by the Water Resources Management Information System [43].

\subsection{Internet of Things (IoT) Monitoring for Harmful Algal Blooms (HABs)}

Fig. 2 describes the pontoon monitoring system consisting of a multi-parameter water quality 74 instrument (EXO-2) and a remote terminal unit (RTU). EXO-2 (YSI Inc., Yellow Springs, Ohio, USA) can measure seven water quality variables: water temperature (WT) $\left({ }^{\circ} \mathrm{C}\right), \mathrm{pH}$, electrical conductivity (EC) (mS/cm), dissolved oxygen (DO) (\%), turbidity (Turb) (FNU), Chl-a ( $\mu \mathrm{g} / \mathrm{L})$ and PC $(\mu \mathrm{g} / \mathrm{L})($ Table S1). The water quality data are collected with RTU (Deongmoon ENT Co., ECO::WATCH RTU V3, Seoul, Korea) on the pontoon and transmitted to a data server through NB-IoT model $[44,45]$. The RTU manages data collection schedule of EXO-2 and power-supply level of pontoon system. The NB-IoT module (SERCOM Co., TPB22-3) is used for low-power

81 and long-distance wireless data communication. Real-time water quality monitoring using 82 pontoon was conducted on the water surface. The water quality sensor of the pontoon was 83 installed from 0.5 to $1.8 \mathrm{~m}$.

\subsection{Chlorophyll-a and Phycocyanin Simulation Using Deep Learning}

86 The deep learning for simulating Chl-a and PC consisted of four steps: (1) preparation of input 87 data (Fig. 3(a)), (2) data preprocessing (Fig. 3(b)), (3) hyperparameters optimization (Fig. 3(c)), 88 and (4) simulation of Chl-a and PC using deep learning (Fig. 3(d)). This study used seven water 89 quality, two hydrological, and two meteorological as input data for simulating Chl-a and PC 90 (Table S1). These hydrological and environmental data have verified the factors to influence 91 algal growth [46]. Prior to application of deep learning, the input data applied three data 
92 preprocessing: min-max, z-score, and robust scaling methods. The min-max method rescales the

93 data set with the range of zero to one using minimum and maximum values [47]. The z-score

94 uses the mean and the standard deviation, thereby the mean value calculates zero. The robust

95 scaling removes the outlier in the dataset and calculates with interquartile range. In addition, this

96 study optimized five hyperparameters using the bayesian optimization algorithm (Table S2). The

97 hyperparameters can control the learning process and backpropagation [48, 49]. Finally, we

98 simulated Chl-a and PC concentrations using three deep learning models: attention LSTM, one-

99 layer LSTM, and two-layer LSTM. In our study, the dataset was randomly assigned to training

100 and validation set from the observation. Previous studies have also used random sampling to

101 divide the training and validation $[50,51]$. Therefore, our dataset was divided into $70 \%$ of

102 training and $30 \%$ of validation by random sampling. Our models have been trained using the

103 adam optimizer to update the model weight and parameters to reduce loss value [52]. We used

104 version 2.10 of Tensorflow API in the Python programming language to build up the deep neural

105 network models [53]. Our model training was performed using an Intel® Core i9-10900F 2.80

106 GHz processor, the DDR4 64 Gigabytes of random-access memory, and NVIDIA GeForce RTX

1073070 graphic card.

108

109 2.3.1. Data preprocessing

110 The environmental variables have high variation with biased or skewed distribution, resulting in

111 the biased model training [54]. These problems cause the presence of outliers, missing values,

112 and non-normal distribution, which has led to a deviation between the input dataset [55, 56]. To

113 solve this problem, this study applied three preprocessing methods: the min-max, z-score, and

114 robust scaling methods. Previous studies demonstrated that the application of data preprocessing 
115 can guarantee the data quality before feeding into the deep learning model to minimize data 116 variability [57]. The min-max linearly transforms original data using minimum and maximum 117 values [58]. The min-max function is expressed as follows:

$$
Y(x)=\frac{x_{i}-\min (x)}{\max (x)-\min (x)}
$$

119 where $Y(x)$ is the normalized value. $x_{i}$ is the data. The $\min (x)$ and $\max (\mathrm{x})$ are minimum and maximum of data. The technique provides the normalized value from zero to one.

121 The z-score transforms the data using the mean and the standard deviation [47]. The z-score is expressed as follows [59]:

$$
Y(x)=\frac{x_{i}-\operatorname{mean}(x)}{\text { standard deviation }(x)}
$$

124 where the mean $(x)$ is the mean of the data and the standard deviation $(x)$ is the standard deviation 125 of data [47].

127 (IQR) that is the difference between the $1^{\text {st }}$ quartile and $3^{\text {rd }}$ quartile, thereby minimizing the 128 impact of outliers [60]. This equation is expressed as:

$$
Y(x)=\frac{x_{i}-Q_{1}(x)}{Q_{3}(x)-Q_{1}(x)}
$$

where $Q_{1}$ is the $1^{\text {st }}$ quartile and $Q_{3}$ is the $3^{\text {rd }}$ quartile.

\subsubsection{Attention LSTM}

133 Attention LSTM is the version of coupled LSTM and attention mechanism (Fig. 4 (a)). In the 134 attention LSTM, the previous information are recurrent to deal with the sequence data by the 135 LSTM layer (Fig. 4(b)). Additionally, this model was combined with the attention mechanism that is known to be used to enhance the model performance and interpretability (Fig. 4(c)) [61]. 
137 The attention mechanism decides the significant part of input data during the model training. In 138 addition, this mechanism can explain the model result by generating the attention score map that 139 can visualize the importance of input data [62]. Vaswani et al. [63] demonstrated that attention140 based models had faster training time than existing recurrent and convolutional neural networks. 141 The following equations are used to calculate the attention mechanism:

$$
\begin{aligned}
e_{t}^{k} & =v_{e}^{t} \cdot \tanh \left(W_{e}\left[h_{t-1} ; s_{t-1}\right]+U_{e} \cdot y^{k}\right) \\
a_{t}^{k} & =\frac{\exp \left(e_{t}^{k}\right)}{\sum_{i=1}^{n} \exp \left(e_{t}^{i}\right)} \\
C_{t} & =\sum_{i=1}^{m} a_{t}^{k} h_{i}
\end{aligned}
$$

145 where the $e_{t}$ and $t$ are alignment score, and the sequence length of input, respectively. $a_{t}^{k}$ is a 146 softmax function that turns an array of alignment scores to sum with one [64]. The parameters $v_{e}$, $147 W_{e}$, and $U_{e}$ are the weight matrices determined by the training process and the $y^{k}$ indicates the 148 number of input data. The $C_{t}$ and $h_{i}$ are context vector and hidden state, respectively.

\subsubsection{Long short-term memory (LSTM)}

151 LSTM is developed based on recurrent neural network (RNN) [65]. The RNN is designed to deal 152 with sequence data by interrelating between the previous state and the current state [66]. The 153 RNN contains a recurrent loop, regulating information to be stored within the network. RNNs are 154 weak to learn the long sequence due to the vanishing gradient problem in the deep neural 155 network which means that previous data is not reflected in the current state [67]. The LSTM is 156 proposed for resolving the vanishing gradient problem by applying gates in RNN cells. The 157 LSTM architecture is composed of three gates namely forget, update, and output gate to regulate 
158 the interaction of the previous information. The LSTM can be calculated by the following 159 equations: regulate the network in the new memory cell, $\bar{c}_{t}$. Then, the current cell state, $c_{t}$, can be updated

171 with the forget and input gate to contain information of current and previous state. $o_{t}$ denotes the 172 activation vectors of the output gate to adjust the output activation of the cell. The $W$ and $b$ 173 indicate weight and bias that can be calculated during the model training. The parameters $h$ and $c$ 174 mean the hidden states and cell states [68].

\subsubsection{Hyper-optimization (HPO)}

177 The hyperparameters have strongly influenced the performance of data-driven models [48]. We 178 obtained the optimal hyperparameter set using the bayesian optimization method [49, 69]. The 179 bayesian optimization algorithm is derivative-free optimization to find the optimal 180 hyperparameters with the gaussian process [70]. The hyperparameter is tuned to minimize the 
181 loss value within the configured range, thereby selecting the parameter to improve the 182 performance of models [71]. In our study, Table S2 describes the hyperparameter range for HPO. 183 The hyperparameters were automatically searched for the optimal value during bayesian 184 optimization process. The mean square error (MSE) was adopted for calculating the loss between 185 simulation and observation [72]. Also, we applied the dropout of 0.3 to prevent the overfitting 186 problem [73]. Libraries of scikit-optimize and Hyperopt were used for HPO [74].

\subsection{Model Evaluation}

189 The model performance was evaluated using the correlation coefficient $(\mathrm{R})$ and root mean square error (RMSE). The R and RMSE can represent the indices for the relationship and error between the observation and the simulation [75]. These indices are obtained using the following equations:

$$
\begin{gathered}
\mathrm{R}=1-\frac{\sum_{t=1}^{n}\left(o_{t}-p_{t}\right)}{\sum_{t=1}^{n}\left(o_{t}-\bar{p}_{t}\right)},-1.0 \leq r \leq 1.0 \\
\mathrm{RMSE}=\sqrt{\frac{\left|\sum_{i=1}^{n}\left(o_{i}-p_{i}\right)^{2}\right|}{n}}
\end{gathered}
$$

where $p_{t}$ is the simulated data, $o_{t}$ is the observed data, $\bar{p}_{t}$ is the mean of the simulated data, and $n$

195 is the number of data. This study adopted the Taylor diagram to visualize the model performance, 196 which can express the geometric relationship [76].

\section{Result and Discussion}

\subsection{Real-time Monitoring for Algal Bloom}

200 The boxplots of Chl-a and PC concentration are presented in Fig. S1. The mean concentrations 201 of Chl-a and PC in Hoenam are $4.47 \mu \mathrm{g} / \mathrm{L}$ and $0.07 \mu \mathrm{g} / \mathrm{L}$, respectively, and those in Chusori were $2029.51 \mu \mathrm{g} / \mathrm{L}$ and $1.32 \mu \mathrm{g} / \mathrm{L}$, respectively. The Chl-a and PC concentrations in Chusori increased 
203 from late summer to autumn, yielding $97.56 \mu \mathrm{g} / \mathrm{L}$ and $31.37 \mu \mathrm{g} / \mathrm{L}$ of peak concentrations, 204 respectively. These concentration levels can be regarded as the 'very bad' level according to 205 ambient water quality standard in South Korea [77]. This was caused by high temperature and 206 excessive nutrient loading by heavy rainfall $[78,79]$. The water temperature in this study ranged 207 from $23^{\circ} \mathrm{C}$ to $31^{\circ} \mathrm{C}$ when $\mathrm{HABs}$ occurred. This range of water temperature can strongly affect 208 the growth rate of algae, the vertical mixture of the freshwater, and the reduction of viscosity 209 [80]. Pawlita-Posmyk et al. [81] referred that the warm water temperature between $15^{\circ} \mathrm{C}$ to $26^{\circ} \mathrm{C}$ 210 can promote algal growth. The peak nutrient concentrations were observed in this bloom period;

211 Hoenam showed TN and TP of $2.59 \mathrm{mg} / \mathrm{L}$ and $0.08 \mathrm{mg} / \mathrm{L}$, respectively, and Chusori showed that

212 of $4.06 \mathrm{mg} / \mathrm{L}$ and $0.20 \mathrm{mg} / \mathrm{L}$. It implies that our study sites received excessive nutrients from the 213 watershed, resulting in the outbreak of cyanobacteria bloom [82]. Paerl et al. [83] demonstrated 214 that the growth of cyanobacteria might have positive relationship with nutrients because this 215 species can use nitrogen and phosphorus to increase biomass.

\section{3.2. Effect of Data Preprocessing}

218 We compared the model performance using the Taylor diagram that can visualize the statistical 219 summary between the observation and simulation (Fig. 5) [84]. Attention LSTM with the z-score 220 showed the highest model performance by having the highest value of $\mathrm{R}$ and the lowest value of 221 RMSE; the average values of R and RMSE were 0.84 and $2.35(\mu \mathrm{g} / \mathrm{L})$, respectively. It implies 222 that the attention LSTM and z-score were suitable to simulate Chl-a and PC. Ding et al. [85] and 223 Luong, Pham and Christopher [61] presented that the attention mechanism improved 224 performance compared to the other models because this mechanism can be useful to capture the 225 feature of input data. Zhang et al. [86] demonstrated that the z-score can stabilize the model 
226 training by reliving the negative effect of the outlier. In the contrast, the 2-layer LSTM and the

227 min-max scaler were improper to simulate HABs, by showing the lowest performance.

228 Especially, the model performance was decreased as increasing the number of layers. It indicates

229 that the complex model might deteriorate the model accuracy than the simple model (i.e., 1-layer

230 LSTM). Cho et al. [87] also showed that the model complexity negatively influenced the model

231 inference, which imposed excessive computation power to identify the important features in data

232 and parameters. Although min-max scaler was popular among preprocessing methods, this

233 method had limited to reduce the effect of outlier and the variation of data [58]. The model

234 performance varied depending on the type of structure and preprocessing. It reveals that the

235 selection of structure and preprocessing method were essential steps for effective model training

236 and application. Chen et al. [88] suggested that the inappropriate selection of them might cause

237 the vanishing gradient, thereby producing worse simulation.

\section{3.3. Hyper-parameter Optimization}

240 Fig. S2 and S3 show the optimization process using the attention LSTM model with z-score

241 scaling. The learning rate is the most sensitive hyperparameter in that the changed slope is the

242 steepest compared to other parameters. During optimization, the learning rate was converged

243 from the large value to the small value, implying that our model preferred the small step size

244 when adjusting the weight and bias. Jang et al. [89] and Yun et al. [90] also recommended the

245 smaller learning rate to simulate the water quality. In addition, the lookback also was the

246 influential factor to the model result. The lookback can define the value how many previous

247 timesteps to simulate the output value [64]. In the contrast, the model performance was weakly

248 influenced by the type of activation functions. Table S3 describes the optimized hyperparameter 
249 value from the optimization process. The optimal batch sizes for Hoenam and Chusori were eight.

250 Previous studies showed that eight of batch size was enough to model training without the

251 vanishing gradient and overfitting problems for the environmental simulation [91]. Our lookback

252 optimal sizes were eight and seven for Hoenam and Chusori, indicating that the HAB simulation

253 required the temporal information from the previous eight and seven days to the current

254 simulation time, respectively.

255

\section{3.4. Chlorophyll a and Phycocyanin simulation}

257 Fig. 6 and 7 present the time series and scatter plot of Chl-a and PC using attention LSTM with

258 z-score scaling. The simulated Chl-a and PC concentrations were similar to the observation in

259 both sites. On the Hoenam, the R and RMSE showed 0.92 and $1.63(\mu \mathrm{g} / \mathrm{L})$ of $\mathrm{Chl}-\mathrm{a}$, and 0.77 and

$2601.66(\mu \mathrm{g} / \mathrm{L})$ of PC, respectively. On the Chusori, the R and RMSE showed 0.82 and $3.61(\mu \mathrm{g} / \mathrm{L})$

261 of $\mathrm{Chl}-\mathrm{a}$, and 0.83 and $2.48(\mu \mathrm{g} / \mathrm{L})$ of $\mathrm{PC}$, respectively. These results implied the acceptable

262 performance and good agreement with the observed Chl-a and PC. In particular, the Chl-a and

263 PC simulation in spring and winter exhibited improved model accuracy compared to the summer

264 season. This is because various external sources (e.g., heavy rainfall, nutrient loading, and warm

265 water) existed that the algae life cycle in the summer season [80]. Park et al. [92] demonstrated

266 that the algae life cycle was significantly influenced by nutrients and discharge from the

267 watershed. The simulated Chl-a concentration showed higher variation than PC concentration

268 from July to October. This is because the concentrations of Chl-a were influenced by the

269 dynamic of diatoms, green algae, and cyanobacteria while PC was an indicator for cyanobacteria

270 that had rapid growth in summer [93]. The Chl-a and PC concentrations in Hoenam had

271 relatively lower concentrations compared to Chusori because Hoenam presented had deep water 
272 above $25 \mathrm{~m}$ compared to Chusori station, resulting in the shorter retention time [37]. Cha et al.

273 [94] reported that a short retention time might restrict algal growth by accelerating the dispersion

274 and advection of HABs.

275

276 3.5. Model Interpretability with Attentions

277 Fig. 8 shows the attention score map to temporally interpret the attention LSTM model. The

278 plots represent the weight of input data to affect the model output [89]. On the attention score

279 map, the color bar indicates the importance of the dataset [64]. The results were highlighted from

280 June to October in Hoenam, indicating that this period was the most influenced period to the

281 model result. In this period, there existed the intensive inflow including the nutrients and warm

282 temperature. Jeong et al. [95] investigated that the HABs have occurred from August to October

283 due to enough nutrients washed from heavy rainfall. Singh et al. [96] also demonstrated that the

284 effect of temperature from $20^{\circ} \mathrm{C}$ to $30^{\circ} \mathrm{C}$ can accelerate algal growth. The highlighted period 285 presented the warm water having the range from $20^{\circ} \mathrm{C}$ to $30^{\circ} \mathrm{C}$, implying that the study sites were 286 appropriate for growing the cyanobacteria. The weight scores of Chusori were highlighted in 287 June. It implies that Chusori was vulnerable to the nutrients source by heavy rainfall compared to 288 temperature because the peak nutrient inflow was observed in June [97]. The lookback from 289 previous six day to the present could be regarded as important factors for simulating Chl-a and 290 PC. The results were related to the initiation for algae developments at a suitable time and 291 inoculum size [98]. Our study was limited to understanding the output by changing the specific 292 input. Further studies would solve this problem by applying dual-stage attention mechanism that 293 can explain the correlation between input and output by extracting the temporal feature of each 294 input [62]. 
1

2

3

4

5

\section{Acknowledgment}

\section{Conclusions}

20

Herein, we implemented LSTM models to simulate the concentrations of Chl-a and PC using IoT monitoring. The real-time investigation of HABs was conducted and these data were then used for model training. Furthermore, we identified the effect of data preprocessing and structure type to model performance. This model was interpreted by analyzing the weight in the attention mechanism. The major findings of this study are as follows:

(1) From the real-time monitoring results, the concentration of Chl-a and PC were peaked in late summer and autumn compared to the other periods.

(2) Attention LSTM with the z-score method showed the highest model performance by having the highest value $\mathrm{R}$ and the lowest value of RMSE; average $\mathrm{R}$ and RMSE values are 0.84 and $2.35(\mu \mathrm{g} / \mathrm{L})$, respectively.

(3) The trained model exhibited that the monitoring data from July to October were highlighted by having the highest weight in the attention mechanism. This implies that this period is the most influenced period to model simulation.

In addressing the water quality problem due to HABs, this study found that the deep learning approach with IoT monitoring had significant potential to detect and quantify HABs with high accuracy. In addition, our approach could utilize alternatives to the traditional water quality modeling by dealing with $\mathrm{HAB}$ variation. Therefore, this study will provide the preliminary information for future deep learning approach in water quality determination. 
1 This work was supported by Electronics and Telecommunications Research Institute(ETRI)

2 grant funded by ICT R\&D program of MSIT/IITP[21HB1310, Space-time complex artificial

3 intelligence blue-green algae prediction technology based on direct-readable water quality

4 complex sensor and hyperspectral image].

5

\section{Author Contributions}

7 D.H.K. (Master student) conducted all modeling and wrote the manuscript. S.M.H. (Master 8 student), A.A. (Ph.D. candidate), and J.C.P. (Ph.D.) assisted manuscript writing. H.K.L. (Ph.D.) 9 supported the experiments. S.S.B. (Ph.D.) and K.H.C. (Professor) revised the manuscript draft. 10 All authors read and approved the final manuscript.

\section{References}

13 [1] Bae S-S, Pyo J, Parchepsky Y, et al. Identification and enumeration of cyanobacteria species 14 using a deep neural network. Ecol. Indic. 2020;115:106395.

15 [2] Luo Y, Yang K, Yu Z, et al. Dynamic monitoring and prediction of Dianchi Lake 16 cyanobacteria outbreaks in the context of rapid urbanization. Environ. Sci. Pollut. Res. $17 \quad 2017 ; 24(6): 5335-5348$.

18 [3] Michalak AM. Study role of climate change in extreme threats to water quality. Nature News. $19 \quad 2016 ; 535(7612): 349$.

20 [4] Joung S-H, Oh H-M, Ko S-R, Ahn C-Y. Correlations between environmental factors and

21 toxic and non-toxic Microcystis dynamics during bloom in Daechung Reservoir, Korea. Harmful 22 Algae. 2011;10(2):188-193. 
1 [5] Cha Y, Park SS, Kim K, Byeon M, Stow CA. Probabilistic prediction of cyanobacteria

2 abundance in a Korean reservoir using a Bayesian Poisson model. Water Resour. Res. $3 \quad 2014 ; 50(3): 2518-2532$.

4 [6] Ingole NP, An K-G. Modifications of nutrient regime, chlorophyll-a, and trophic state 5 relations in Daechung Reservoir after the construction of an upper dam. J. Ecol. Environ. $6 \quad 2016 ; 40(1): 1-10$.

7 [7] Barruffa AS, Pardo Á, Faggian R, Sposito V. Monitoring cyanobacterial harmful algal

8 blooms by unmanned aerial vehicles in aquatic ecosystems. Environ. Sci.: Water Res. Technol. $9 \quad 2021 ; 7(3): 573-583$.

10 [8] Lekki J, Deutsch E, Sayers M, et al. Determining remote sensing spatial resolution 11 requirements for the monitoring of harmful algal blooms in the Great Lakes. J. Great Lakes Res. $12 \quad 2019 ; 45(3): 434-443$.

13 [9] Lee JHW, Hodgkiss IJ, Wong K, Lam I. Real time observations of coastal algal blooms by an 14 early warning system. Estuar. Coast. Shelf Sci. 2005;65(1-2):172-190.

15 [10] Barruffa AS, Pardo Á, Faggian R, Sposito V. Monitoring cyanobacterial harmful algal 16 blooms by unmanned aerial vehicles in aquatic ecosystems. Environ. Sci.: Water Res. Technol. $17 \quad 2021 ; 7(3): 573-583$.

18 [11] Geetha S, Gouthami S. Internet of things enabled real time water quality monitoring system. 19 Smart Water. 2016;2(1):1-19.

20 [12] Carpenter CM, Wong LYJ, Gutema DL, Helbling DE. Fall Creek Monitoring Station: using 21 environmental covariates to predict micropollutant dynamics and peak events in surface water 22 systems. Environ. Sci. Technol. 2019;53(15):8599-8610. 
1 [13] Cho K, Pachepsky Y, Ligaray M, Kwon Y, Kim KH. Data assimilation in surface water

2 quality modeling: A review. Water Res. 2020;186(1):116307.

3 [14] Wong BP, Kerkez B. Real-time environmental sensor data: An application to water quality

4 using web services. Environ. Model. Softw. 2016;84:505-517.

5 [15] Hu Z, Zhang Y, Zhao Y, et al. A water quality prediction method based on the deep LSTM

6 network considering correlation in smart mariculture. J. Sens. 2019;19(6):1420.

7 [16] Pyo, J., Cho, K. H., Kim, K, et al. Cyanobacteria cell prediction using interpretable deep

8 learning model with observed, numerical, and sensing data assemblage. Water Res. $9 \quad 2021 ; 203: 117483$.

10 [17] Hamrick JM. A three-dimensional environmental fluid dynamics computer code:

11 Theoretical and computational aspects. 1992. p. 7-8.

12 [18] Arnold JG, Srinivasan R, Muttiah RS, Williams JR. Large area hydrologic modeling and 13 assessment part I: model development 1. J. Am. Water Resour. Assoc. 1998;34(1):73-89.

14 [19] Cole TM, Buchak EM. CE-QUAL-W2: A Two-Dimensional, Laterally Averaged, 15 Hydrodynamic and Water Quality Model, Version 2.0. User Manual. US Army Engineering and 16 Research Development Center, Vicksburg, MS.; 1995. p. 1-2.

17 [20] Byun J-H, Cho I-H, Hwang S-J, et al. Relationship between a dense bloom of 18 cyanobacterium Anabaena spp. and rainfalls in the North Han River system of South Korea. 19 Korean J. Ecol. Environ. 2014;47(2):116-126.

20 [21] Nishu SD, Kang Y, Han I, Jung TY, Lee TK. Nutritional status regulates algicidal activity 21 of Aeromonas sp. L23 against cyanobacteria and green algae. PLoS One. 2019;14(3):e0213370. 
1 [22] Kim S, Kwon YS, Pyo J, et al. Developing a Cloud-based Toolbox for Sensitivity Analysis

2 of a Water Quality Model. Environ. Model. Softw. 2021;141:105068.

3 [23] LeCun Y, Bengio Y, Hinton G. Deep learning. Nature 2015;521(7553):436-444.

4 [24] GERS, Felix A.; SCHMIDHUBER, Jürgen, Fred. Learning to forget: Continual prediction 5 with LSTM. Neural Comput. 2000;12(10):2451-2471.

6 [25] Baek S-S, Pyo J, Chun JA. Prediction of Water Level and Water Quality Using a CNN-

$7 \quad$ LSTM Combined Deep Learning Approach. Water 2020;12(12):3399.

8 [26] Zhang D, Lindholm G, Ratnaweera H. Use long short-term memory to enhance Internet of

9 Things for combined sewer overflow monitoring. J. Hydrol. 2018;556:409-418.

[27] Castelvecchi D. Can we open the black box of AI?. Nature News. 2016;538(7623):20.

11 [28] Lee T, Singh VP, Cho KH. Deep Learning for Hydrometeorology and Environmental 12 Science. Springer; 2021. p. 21-25.

13 [29] Zheng X, Wang M, Ordieres-Meré J. Comparison of data preprocessing approaches for 14 applying deep learning to human activity recognition in the context of industry 4.0. J. Sens. $15 \quad 2018 ; 18(7): 2146$.

16 [30] Shen Y, Ma Y, Deng S, Huang C-J, Kuo P-H. An ensemble model based on deep learning 17 and data preprocessing for short-term electrical load forecasting. Sustainability 2021;13(4):1694.

18 [31] Carpenter SR, Cole JJ, Pace ML, et al. Early warnings of regime shifts: a whole-ecosystem 19 experiment. Science 2011;332(6033):1079-1082.

20 [32] Boyer JN, Kelble CR, Ortner PB, Rudnick DT. Phytoplankton bloom status: Chlorophyll a 21 biomass as an indicator of water quality condition in the southern estuaries of Florida, USA. Ecol. 22 Indic. 2009;9(6):S56-S67. 
1 [33] Wang $\mathrm{Y}$, Huang $\mathrm{M}$, Zhu $\mathrm{X}$, et al. Attention-based LSTM for aspect-level sentiment

2 classification. Proceedings of the 2016 conference on empirical methods in natural language

3 processing; 2016. p. 606-615.

4 [34] Shin J, Cho K, Oh I. Dynamics of water environmental factors and phytoplankton in 5 Taechong Reservoir. Korean J. Environ. Biol. 1999;17(4):529-541.

6 [35] Moo Joon S, Jae Yong Y, Soo Hyung L. Water Quality Properties of Tributaries of

7 Daechung Lake, Korea. Korean J. Ecol. Environ. 2015;48(1):12-25.

8 [36] Kwon YS, Pyo J, Kwon Y-H, et al. Drone-based hyperspectral remote sensing of 9 cyanobacteria using vertical cumulative pigment concentration in a deep reservoir. Remote Sens. Environ. 2020;236:111517.

11 [37] Pyo J, Kwon YS, Ahn JH, et al. Sensitivity Analysis and Optimization of a Radiative 12 Transfer Numerical Model for Turbid Lake Water. Int. J. Remote Sens. 2021;13(4):709.

13 [38] Bae D-Y, Yang E-C, Jung S-H, Lee J-H, An K-G. Nutrients and chlorophyll dynamics 14 along the longitudinal gradients of Daechung reservoir. Korean J. Ecol. Environ.2007;40(2):28515293.

16 [39] Pyo J, Kwon YS, Ahn J-H, et al. Sensitivity Analysis and Optimization of a Radiative 17 Transfer Numerical Model for Turbid Lake Water. Int. J. Remote Sens. 2021;13(4):709.

18 [40] Jang M, Seo D, Kim J, Kim J, et al. Spatiotemporal algal bloom prediction of geum river, 19 Korea using the deep learning models in company with the EFDC model. Proceedings of the 202020 Summer Simulation Conference; 2020 p. 1-11.

21 [41] National Institute of Environmental Research (NIER). Ministry of Environment Korea 22 [cited 28 October 2021]. Available from: http://water.nier.go.kr. 
1 [42] Korea Meteorological Administration (KMA). Ministry of Environment Korea [cited 28

2 October 2021]. Available from: https://data.kma.go.kr/.

3 [43] Water Resources Management Information System (WAMIS). Han River Flood Control

4 Office [cited 28 October 2021]. Available from: http://www.wamis.go.kr/.

5 [44] Sinha RS, Wei Y, Hwang S-H. A survey on LPWA technology: LoRa and NB-IoT. Ict.

6 Express. 2017;3(1):14-21.

7 [45] Huan J, Li H, Wu F, Cao W. Design of water quality monitoring system for aquaculture

8 ponds based on NB-IoT. Aquac. Eng. 2020;90:102088.

9 [46] Brussaard CP, Riegman R. Influence of bacteria on phytoplankton cell mortality with 10 phosphorus or nitrogen as the algal-growth-limiting nutrient. Aquat. Microb. Ecol. $11 \quad$ 1998;14(3):271-280.

12 [47] Shuai Y, Zheng Y, Huang H, et al. Hybrid Software Obsolescence Evaluation Model Based on PCA-SVM-GridSearchCV. 2018 IEEE 9th International Conference on Software Engineering 14 and Service Science (ICSESS); 2018: IEEE. p. 449-453.

15 [48] Hutter F, Lücke J, Schmidt-Thieme L. Beyond manual tuning of hyperparameters. KI $16 \quad$ Kunstl. Intell. 2015;29(4):329-337.

17 [49] Jones DR, Schonlau M, Welch WJ. Efficient global optimization of expensive black-box 18 functions. J. Glob. Optim. 1998;13(4):455-492.

19 [50] Caruana R, Niculescu-Mizil A. An empirical comparison of supervised learning algorithms.

20 Proceedings of the 23rd international conference on Machine learning; 2006. p. 161-168.

21 [51] Sechidis K. Comparison of different preprocessing techniques and feature selection 22 algorithms in cancer datasets. Front. Genet. 2021;12:1-17. 
1 [52] Kingma DP, Ba JL. ADAM: A Method for Stochastic Optimization. In: 3rd International

2 Conference on Learning Representations, ICLR; 7-9 May 2015; San Diego, CA. Ithaca, NY:

3 arXiv.org; 2015.

4 [53] Abadi, Martín, Barham, et al. Tensorflow: Large-scale machine learning on heterogeneous

5 distributed systems. arXiv preprint arXiv: 1603.04467; 2016.

6 [54] Fu L, Wang Y-G. Statistical tools for analyzing water quality data. Environ. Monit. Assess. $7 \quad 2012 ; 1: 143-168$.

8 [55] Wang W, Vrijling J, Van Gelder PH, Ma J. Testing for nonlinearity of streamflow processes 9 at different timescales. J. Hydrol. 2006;322(1-4):247-268.

10 [56] Pang G, Shen C, Cao L, Hengel AVD. Deep Learning for Anomaly Detection: A Review. 11 ACM Comput. Surv. 2021;54(2):1-38.

12 [57] Nayak S, Misra BB, Behera HS. Impact of data normalization on stock index forecasting. 13 Int. J. Comput. Inf. Syst. Ind. Manag. Appl. 2014;6:257-269.

14 [58] García S, Luengo J, Herrera F. Data preprocessing in data mining: Springer Sci. Rev. 2015. 15 p. $46-47$.

16 [59] Luong M-T, Pham H, Manning CD. Effective approaches to attention-based neural machine 17 translation. arXiv preprint arXiv:1508.04025; 2015.

18 [60] Vaitheeshwari R, SathieshKumar V. Performance analysis of epileptic seizure detection 19 system using neural network approach. In: 2019 International Conference on Computational 20 Intelligence in Data Science (ICCIDS); $21-23$ February 2019; Chennai, India. IEEE; 2019. p. 1-5. 21 doi: 10.1109/ICCIDS.2019.8862158. 
1 [61] Luong M-T, Pham H, Christopher. Effective Approaches to Attention-based Neural

2 Machine Translation. arXiv pre-print server. 2015.

3 [62] Qin Y, Song D, Chen H, et al. A dual-stage attention-based recurrent neural network for

4 time series prediction. arXiv preprint arXiv:1704.02971; 2017.

5 [63] Vaswani A, Shazeer N, Parmar N, et al. Attention is all you need. arXiv preprint arXiv:

$6 \quad 1706.03762 .2017$.

7 [64] Chollet F. Deep learning with Python. New York: Manning; 2017. p. 25-55

8 [65] Sherstinsky A. Fundamentals of recurrent neural network (RNN) and long short-term

9 memory (LSTM) network. Physica D. 2020;404:132306.

10 [66] Rumelhart DE, Hinton GE, Williams RJ. Learning representations by back-propagating 11 errors. Nature 1986;323(6088):533-536.

12 [67] Hochreiter S. The vanishing gradient problem during learning recurrent neural nets and 13 problem solutions. Int. J. Uncertain. Fuzziness Knowl.-Based Syst. 1998;6(02):107-116.

14 [68] Abbas A, Baek S, Kim M, et al. Surface and sub-surface flow estimation at high temporal 15 resolution using deep neural networks. J. Hydrol. 2020;590:125370.

16 [69] Mockus J, Tiesis V, Zilinskas A. The application of Bayesian methods for seeking the 17 extremum. J. Glob. Optim. 1978;2(117-129):2.

18 [70] Bergstra J, Bardenet R, Bengio Y, Kégl B, et al. Algorithms for hyper-parameter 19 optimization. Advances in neural information processing systems, 2011, 24.

20 NIPS'11: Proceedings of the 24th International Conference on Neural Information Processing 21 SystemsDecember 2011 Pages 2546-2554

22 
1 [71] Santner TJ, Williams BJ, Notz WI, Williams BJ. The design and analysis of computer 2 experiments: Springer; 2003.

3 [72] Heinermann J, Kramer O. Machine learning ensembles for wind power prediction. Renew. $4 \quad$ Energy. 2016;89:671-679.

5 [73] Srivastava N, Hinton G, Krizhevsky A, Sutskever I, Salakhutdinov R. Dropout: a simple 6 way to prevent neural networks from overfitting. J Mach Learn Res. 2014;15(1):1929-1958.

7 [74] Bergstra J, Komer B, Eliasmith C, Yamins D, Cox DD. Hyperopt: a python library for 8 model selection and hyperparameter optimization. Comput. Sci. Discov. 2015;8(1):014008.

9 [75] Taylor R. Interpretation of the correlation coefficient: a basic review. J. Diagn. Med. $10 \quad 1990 ; 6(1): 35-39$.

11 [76] Taylor KE. Summarizing multiple aspects of model performance in a single diagram. 12 Journal of Geophysical Research: J. Atmos. 2001;106(D7):7183-7192.

13 [77] Lim D, Lee Y, Kim K. Evaluation of Water Quality Characteristics and Ecosystem Health 14 of Yongpung Reservoir, Korean J. Environ. Health Sci. 2019;45(1):42-53.

15 [78] Hee-Mock O, Kim D-H. Short-term prediction of the blue-green algal bloom in Daechung 16 Reservoir. Korean Journal of Limnology. 1995;28(2):127-135.

17 [79] Oh K-C, Hee-Mock O, Lee J-H, Maeng J-S. The diurnal vertical migration of phytoplankton 18 in Daechung Reservoir. Korean Journal of Limnology. 1995;28(4):437-446.

19 [80] Joehnk KD, Huisman J, Sharples J, et al. Summer heatwaves promote blooms of harmful 20 cyanobacteria. Glob. Change Biol. Bioenergy. 2008;14(3):495-512. 
[81] PAWLITA-POSMYK, Monika; WZOREK, Małgorzata; PŁACZEK, Małgorzata. The

2 influence of temperature on algal biomass growth for biogas production. In: MATEC Web of 3 Conferences. EDP Sciences, 2018. p. 04008.

4 [82] Feng T, Wang C, Wang P, Qian J, Wang X. How physiological and physical processes 5 contribute to the phenology of cyanobacterial blooms in large shallow lakes: A new Euler$6 \quad$ Lagrangian coupled model. Water Res. 2018;140:34-43.

7 [83] Paerl HW, Xu H, McCarthy MJ, et al. Controlling harmful cyanobacterial blooms in a 8 hyper-eutrophic lake (Lake Taihu, China): the need for a dual nutrient (N \& P) management $9 \quad$ strategy. Water Res. 2011;45(5):1973-1983.

[84] Barzegar R, Aalami MT, Adamowski J. Short-term water quality variable prediction using a 11 hybrid CNN-LSTM deep learning model. Stoch. Environ. Res. Risk Assess. 2020:1-19.

12 [85] Ding Y, Zhu Y, Feng J, Zhang P, Cheng Z. Interpretable spatio-temporal attention LSTM model for flood forecasting. Neurocomputing. 2020;403:348-359.

14 [86] Zhang J, Zhu Y, Zhang X, Ye M, Yang J. Developing a Long Short-Term Memory (LSTM)

15 based model for predicting water table depth in agricultural areas. J. Hydrol. 2018;561:918-929.

16 [87] Cho H, Park H, et al. Merged-LSTM and multistep prediction of daily chlorophyll-a 17 concentration for algal bloom forecast. In: IOP Conference Series: Earth and Environmental 18 Science. IOP Publishing, 2019. p. 012020.

19 [88] Chen T, Honda K. Solving data preprocessing problems in existing location-aware systems.

20 J. Ambient Intell. Humaniz. Comput. 2018;9(2):253-259.

21 [89] Jang J, Abbas A, Kim M, et al. Prediction of antibiotic-resistance genes occurrence at a 22 recreational beach with deep learning models. Water Res. 2021:117001. 
1 [90] Yun D, Abbas A, Jeon J, et al. Developing a deep learning model for the simulation of

2 micro-pollutants in a watershed. J. Clean. Prod. 2021;300:126858.

3 [91] Zhang J, Wang X, Zhao C, et al. Application of cost-sensitive LSTM in water level

4 prediction for nuclear reactor pressurizer. Nucl. Eng. Technol. 2020;52(7):1429-1435.

5 [92] Park H-K, Lee H-J, Heo J, et al. Deciphering the key factors determining spatio-temporal

6 heterogeneity of cyanobacterial bloom dynamics in the Nakdong River with consecutive large

7 weirs. Sci. Total Environ. 2021;755:143079.

8 [93] Graham JL, Loftin KA. CYANOBACTERIA IN LAKES AND 7.5 RESERVOIRS: TOXIN

$9 \quad$ AND TASTE-AND-ODOR SAMPLING GUIDELINES. 2008.

[94] Cha Y, Cho KH, Lee H, Kang T, Kim JH. The relative importance of water temperature and 11 residence time in predicting cyanobacteria abundance in regulated rivers. Water Res. $12 \quad 2017 ; 124: 11-19$.

13 [95] Jeong D-H, Lee J, Kim K, et al. A study on the management and improvement of alert 14 system according to algal bloom in the Daecheong Reservoir. J. Environ. Impact Assess. $15 \quad 2011 ; 20(6): 915-925$.

16 [96] Singh S, Singh P. Effect of temperature and light on the growth of algae species: a review.

17 Renew. Sust. Energ. Rev. 2015;50:431-444.

18 [97] Lee J, Yoon J, Choi I, et al. Vertical Distribution of Harmful Cyanobacterial in the 19 Daechung Reservoir. J. Korean Soc. Water Environ. 2016;1:464-465.

20 [98] Davis KE, Joseph SJ, Janssen PH. Effects of growth medium, inoculum size, and incubation 21 time on culturability and isolation of soil bacteria. Appl. Environ. Microbiol. 2005;71(2):826-834. 

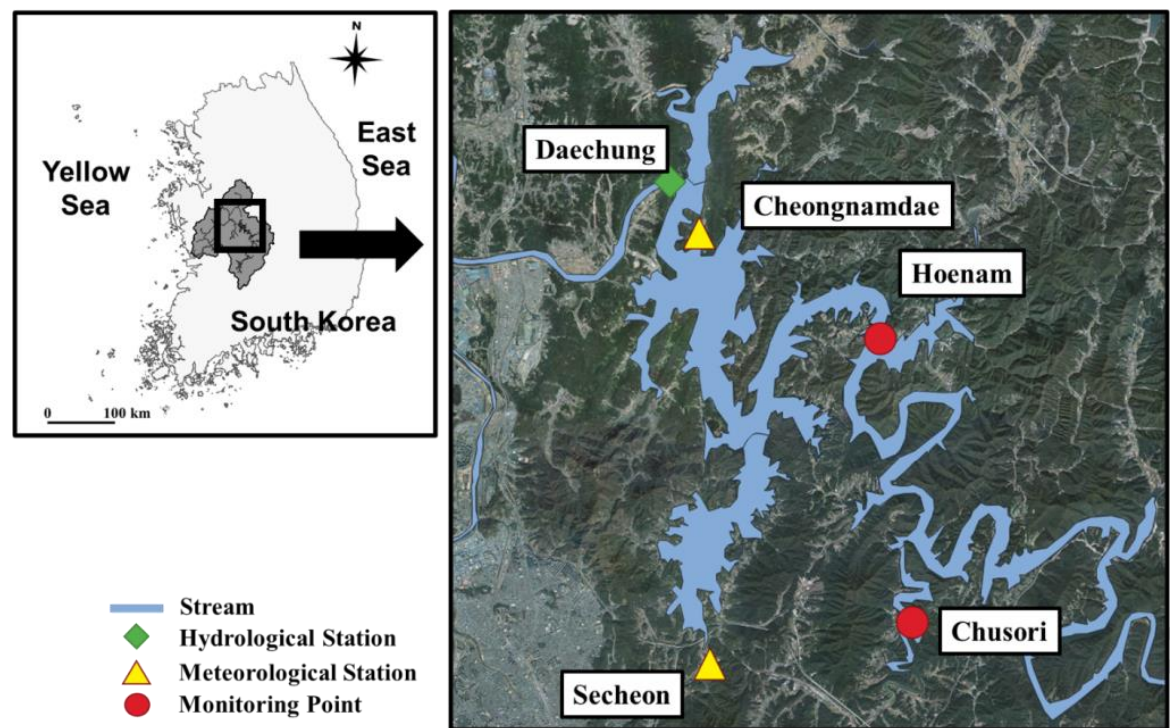

Secheon

2 Fig. 1. Study area: Daechung reservoir with hydrological stations, meteorological stations, and 3 monitoring points. Green diamond, yellow square, and red circle indicate the hydrological station, 4 the meteorological station, and the monitoring point, respectively.

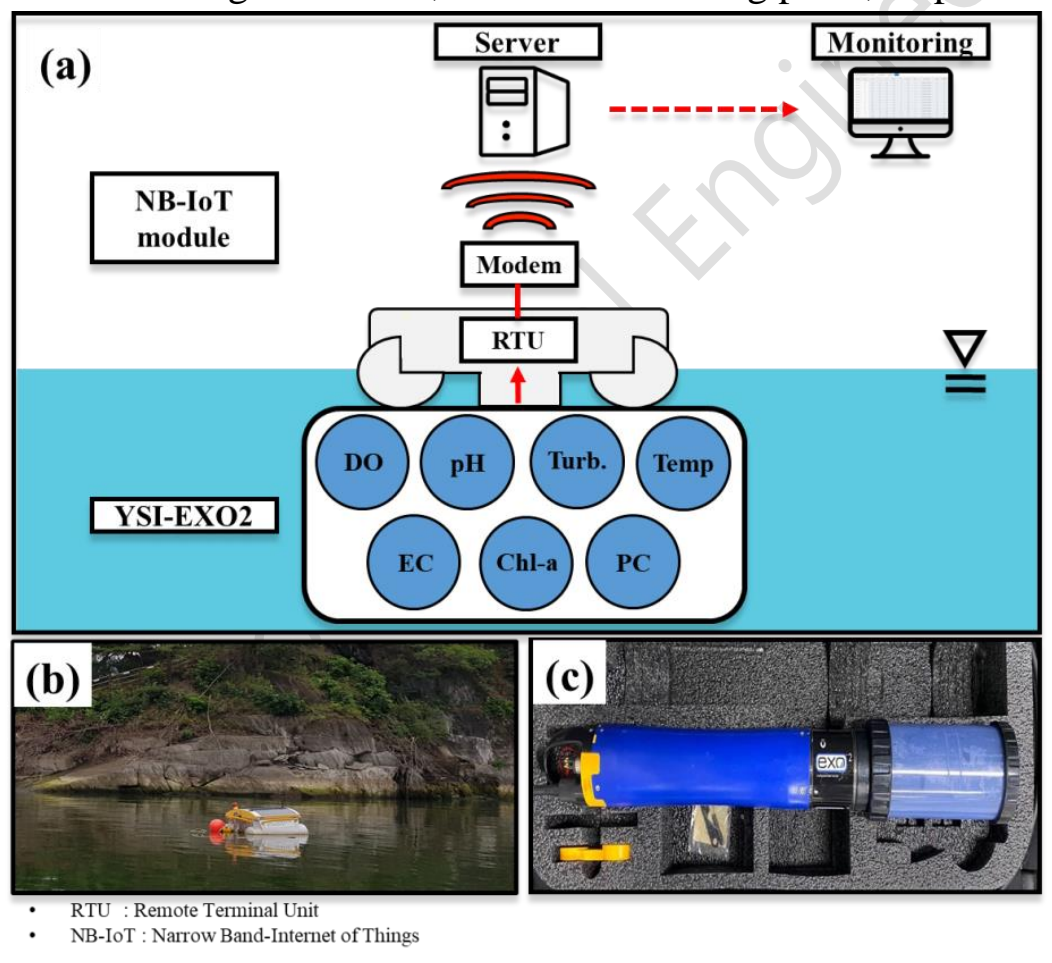

6 Fig. 2. Pontoon monitoring system in Daechung Reservoir. (a) pontoon monitoring system for measuring water quality variables; (b) pontoon monitoring system in study site; (c) YSI-EXO-2 multi-parameter water quality instrument. 


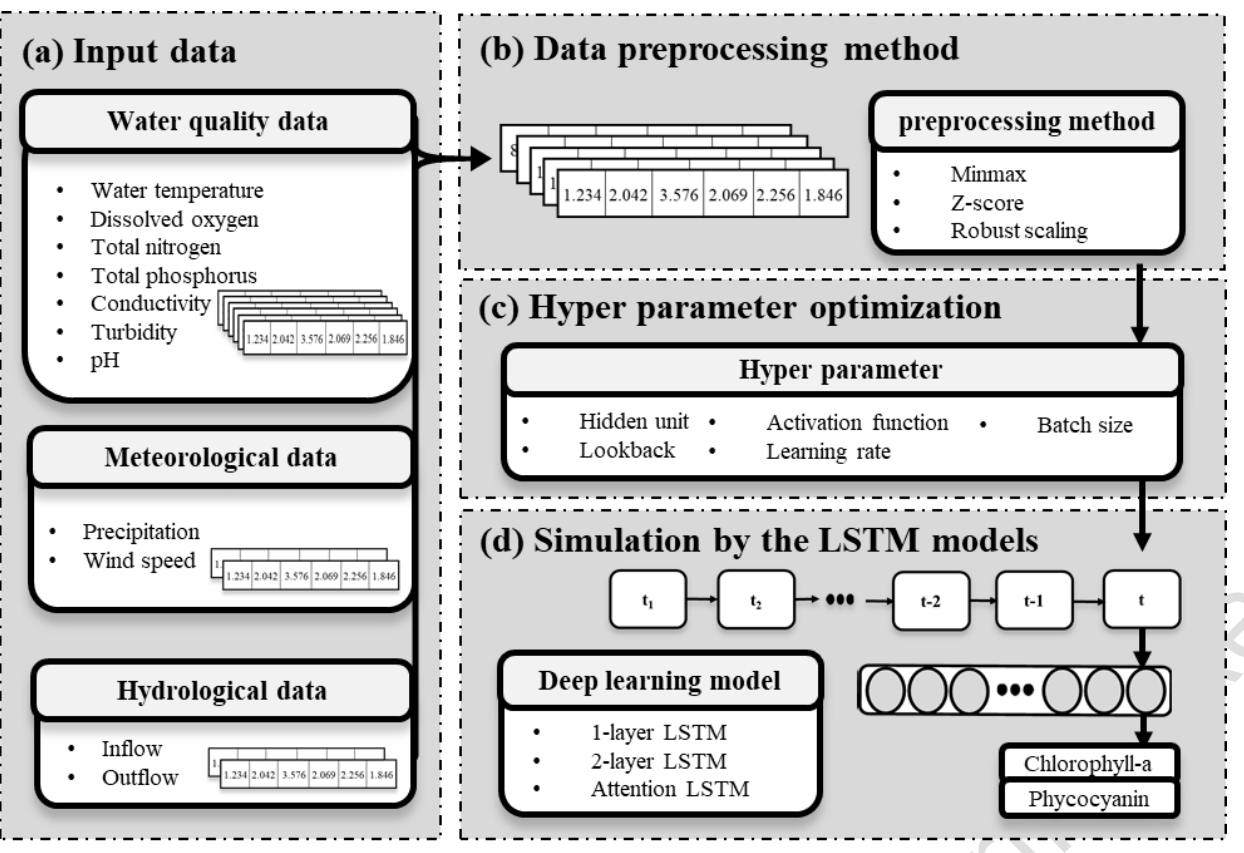

Fig. 3. Schematic diagram for simulating Chl-a and PC concentration.
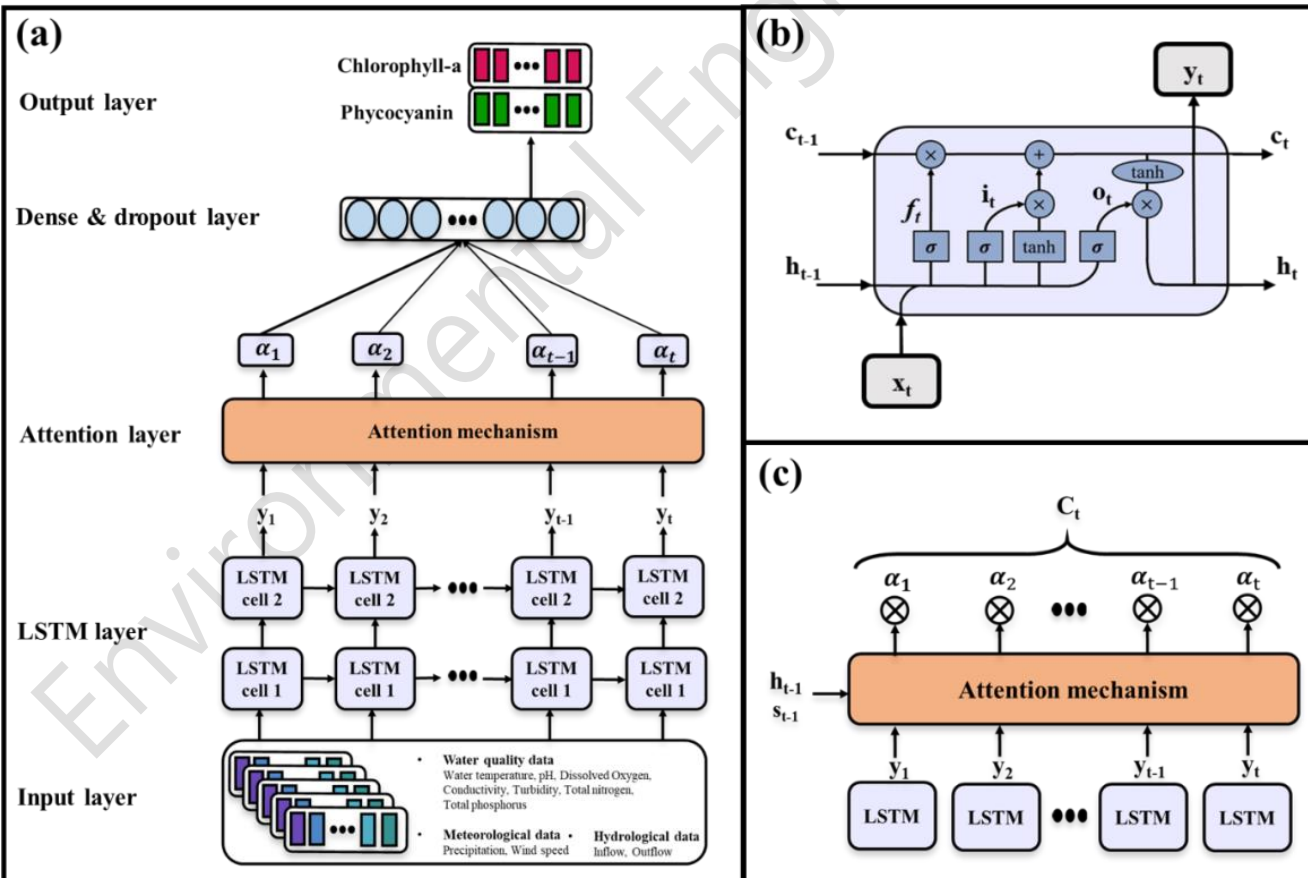

Fig. 4. Descriptions of LSTM and attention LSTM; (a) attention LSTM, (b) LSTM mechanism, and (c) attention mechanism. 
Hoenam
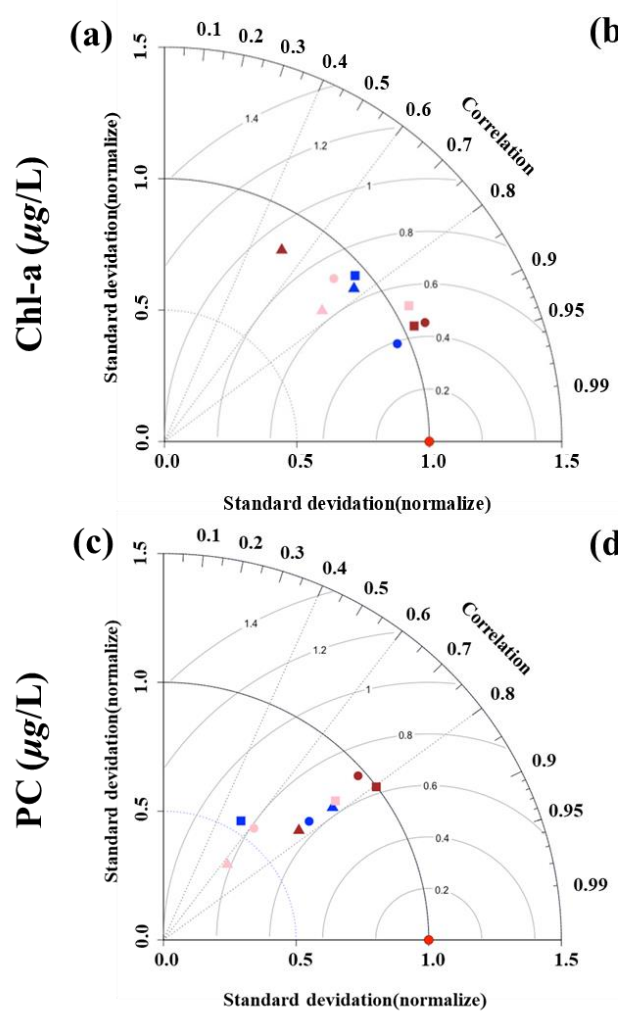

Chusori

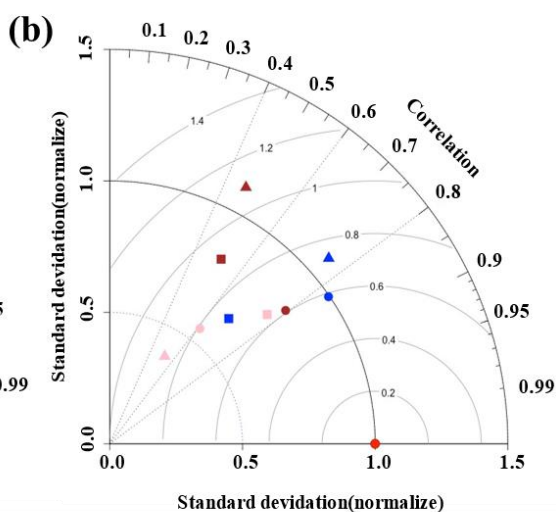

(d)

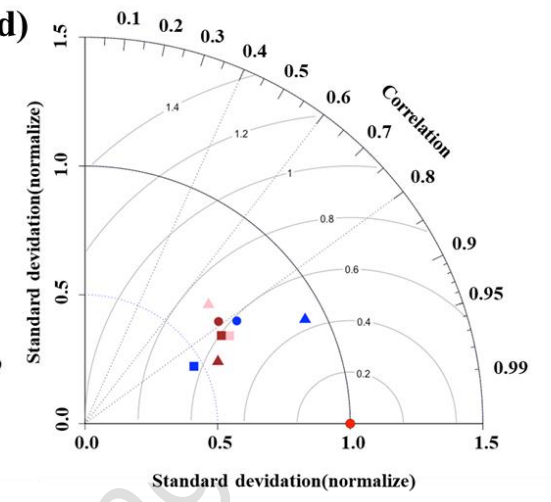

Observation 1 Layer-LSTM

2 Layer-LSTM

Attention-LSTM

$\square$ Min-max

$\bigcirc$ Z-Score

$\triangle$ Robust scaling

Fig. 5. Taylor plots of the LSTM models including correlation coefficient, normalized standard

3 deviation, and centered pattern RMSE. Red, brown, pink, and blue color indicate observation, 14 layer LSTM, 2-layer LSTM, and attention LSTM, respectively, while square, circle, and triangle 5 shapes indicate min-max, z-score, and robust scaling method, respectively. 

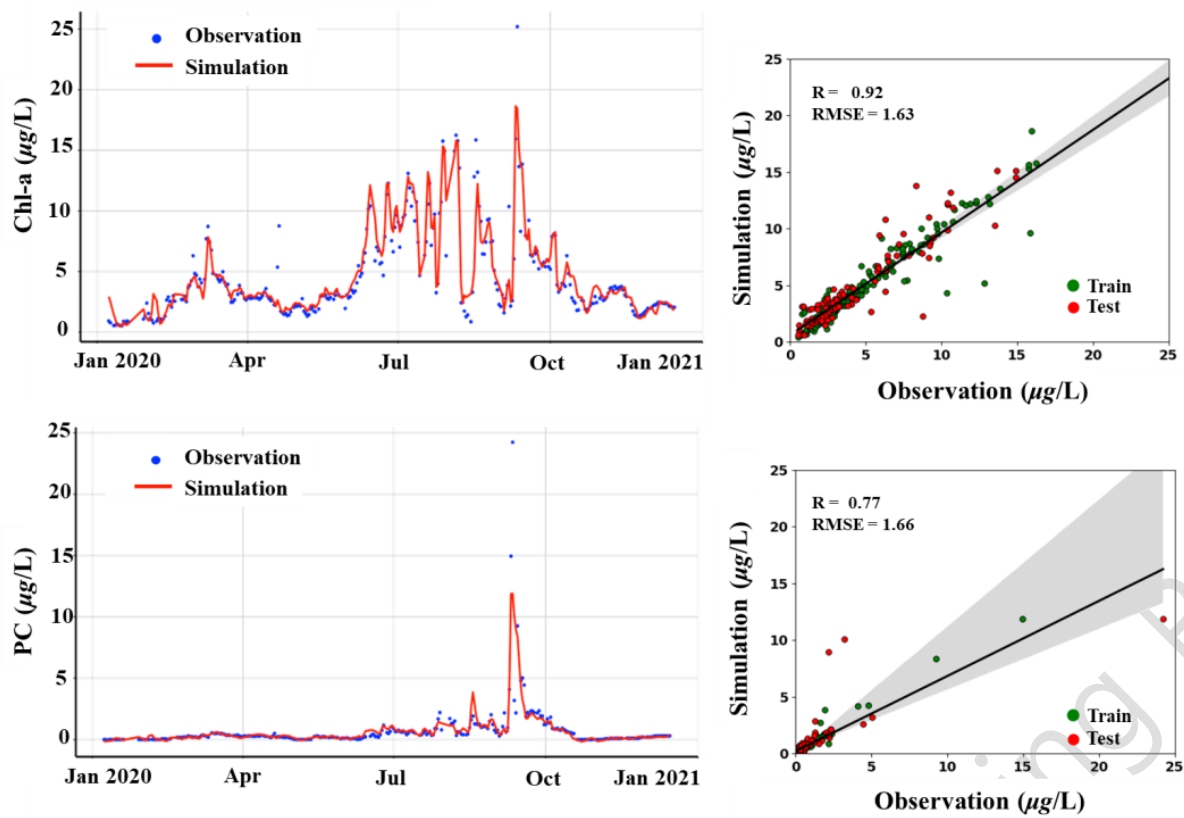

Fig. 6. Comparison of simulated Chl-a and PC concentrations in Hoenam with observation.
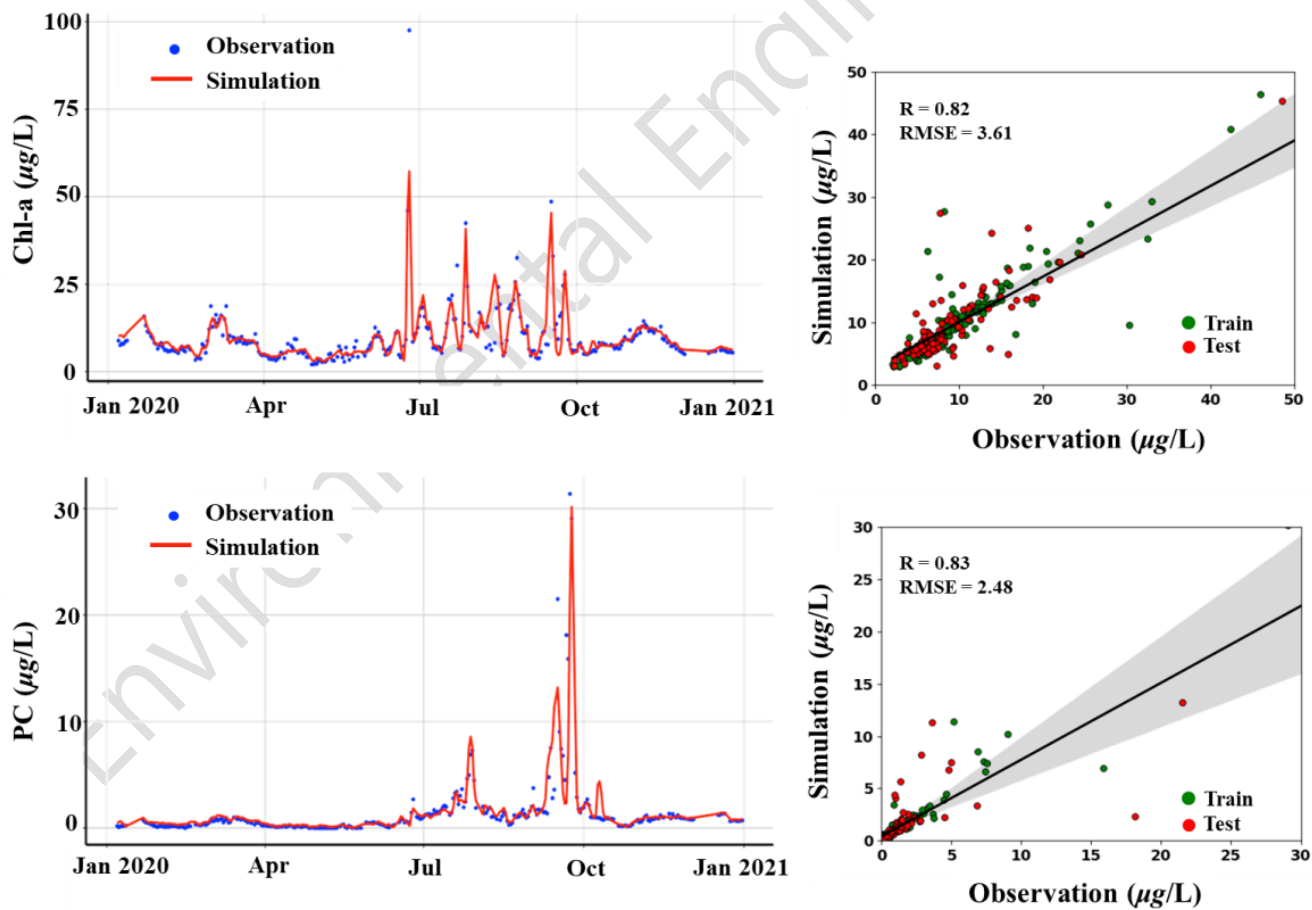

Fig. 7. Comparison of simulated Chl-a and PC concentrations in Chusori with observation. 



1

2 Fig. 8. Attention score maps of the attention LSTM model. The color bar indicates the 3 importance of the dataset.

4 